
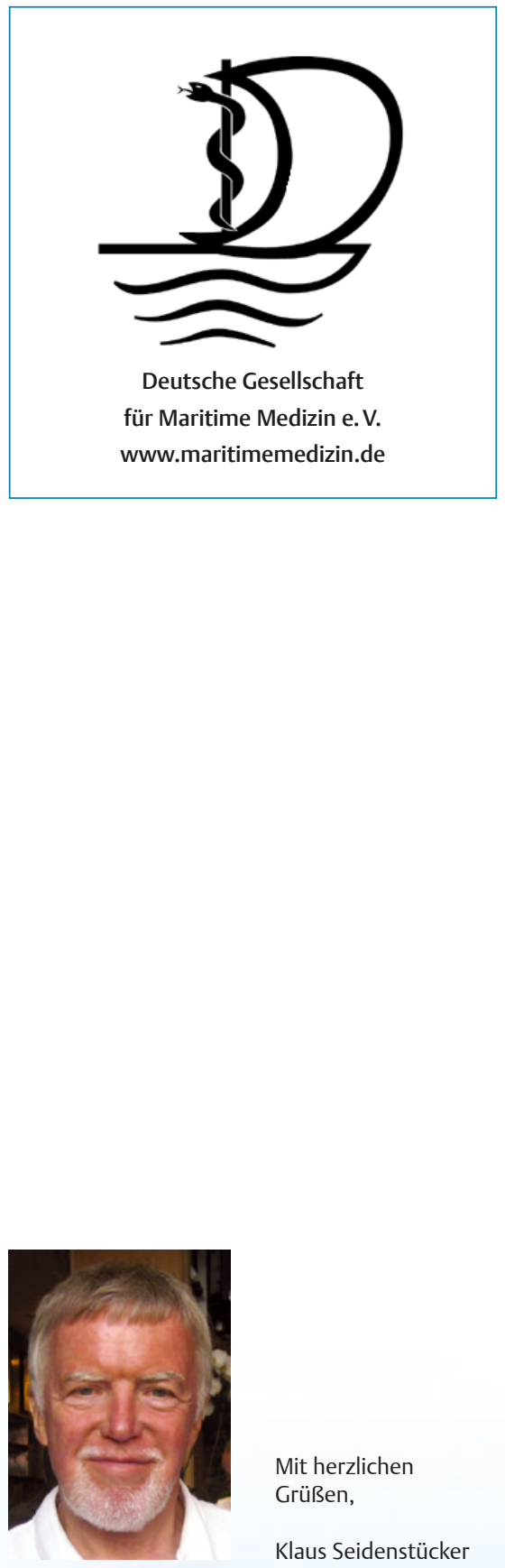

Mit herzlichen

Grüßen,

Klaus Seidenstücker

\title{
Liebe Mitglieder,
}

wenn Sie diese Zeilen ins Haus bekommen, dann haben wir unsere Mitgliederversammlung hinter uns und hoffentlich die erforderlichen Weichen gestellt, um unserer Gesellschaft eine lebendige $\mathrm{Zu}$ kunft zu gestalten.

Eines der bedeutsamsten Vorhaben ist sicher unsere Bewerbung für die Ausrichtung des International Symposium on Maritime Health 2019 zusammen mit dem Hamburger Zentralinstitut für Arbeitsmedizin und Maritime Medizin.

Der Erscheinungstermin dieser Ausgabe koinzidiert mit dem Entscheidungstermin des IMHA-Vorstands, ob wir als, local organizer ausgewählt werden. 30 Jahre nach dem ,First European Nautical Medical Meeting،, welches den Grundstein für die Geburt und Entwicklung der International Maritime Health Association (IMHA) legte, wäre dann Hamburg wieder Austragungsort der wohl renommiertesten maritimmedizinischen Fortbildungsveranstaltung.

Die Aufgabe und die damit verbundene Arbeit sind nicht zu unterschätzen und wir werden Ihre Bereitschaft brauchen, uns dabei nach Kräften zu unterstützen. Schon jetzt gilt es, das Ziel ins Auge zu fassen, unseren Vorstand so zu besetzen, dass ausreichende Zeit und Arbeitskraft für die 2-jährige Vorbereitungsphase und am Ende für die Durchführung des Symposiums zur Verfügung stehen. Ich sage das nicht ohne Grund und aus den Erfahrungen der letzten Jahre. Es geht eben nicht nur darum, ein Amt inne zu haben sondern vor allem darum, dessen Aufgaben sachgerecht zu erfüllen! Ein Jeder prüfe sich also, wieviel freie Valenzen ihm für die Wahrnehmung zusätzlicher Pflichten zur Verfügung stehen. Darüber steht aber auch die Prüfung, wie sehr ihm die maritime Medizin am Herzen liegt. Und ich hoffe sehr, dass die Resultante deutlich über die Bereitschaft zum Zahlen eines Beitrags und die passive Rezeption von Information hinausgeht!

Ende Januar 2017 werden wir einen neuen Vorstand zu wählen haben und dabei die Voraussetzungen schaffen müssen, um das Wagnis 2019 erfolgreich bestehen zu können. Überlegen Sie alle, wozu Sie gegebenenfalls bereit sind.

Soweit zu den Fernzielen. Im Nahbereich planen wir - voraussichtlich im Mai einen Workshop mit Kolleginnen und Kollegen, die in der medizinischen Betreuung der Binnenschifffahrt engagiert sind.

Die IMHA hat leise angefragt, ob wir einen Folgeworkshop zum Thema der medizinischen Vorsorge und Betreuung von transozeanischen Segelrennen gestalten können. Das wäre vorzugsweise in der Nachbarschaft unserer größeren Segelereignisse zu planen.

Im Oktober/November überlegen wir eine Neuauflage unseres Einweisungsseminars in die maritime Medizin.

Und hinweisen möchte ich noch auf den alljährlichen Offshoreworkshop, auch wenn der nicht mehr unter dem Schirm der DGMM stattfindet.

Also beobachten Sie den Veranstaltungskalender auf unserer Internetseite www.maritimemedizin.de und, save the dates'! 


\section{AG Maritime Notfallmedizin der DGMM}

Der Vorstand der Deutschen Gesellschaft für Maritime Medizin e.V. (DGMM) hat in einer seiner letzten Sitzungen auf Anregung aus dem Kreis der Mitglieder die Gründung einer Arbeitsgruppe mit dem Titel „Maritime Notfallmedizin“ beschlossen. Diese soll hier vorgestellt und der Hintergrund erläutert werden.

\section{Hintergrund}

Die Rettung und notfallmedizinische Versorgung auf See stellt eine besondere Herausforderung dar. Rettungseinheiten, die in diesem Umfeld operieren, müssen sich mit den extrem widrigen Umweltbedingungen und großen logistischen Herausforderungen auseinandersetzen. Schon dadurch unterscheidet sich die maritime Notfallmedizin ganz wesentlich von der „normalen“ Notfallmedizin an Land.

Besonderheiten der maritimen

Notfallmedizin

Eine ganz wesentliche Besonderheit der maritimen Notfallmedizin sind die bei Notfällen verlängerten Intervalle der Erstversorgung, Behandlung und Beförderung in die Klinik. Kernelement der maritimen Notfallversorgung auf Handelsschiffen ist daher die telemedizinisch angeleitete Laienhilfe zur Überbrückung dieser Zeiträume.

Auf Kreuzfahrtschiffen hingegen ist regelhaft ärztliches Personal anwesend, sodass die medizinische Notfallversorgung hier unter gänzlich anderen Rahmenbedingungen stattfindet. Hinzu kommt, dass Kreuzfahrtschiffe häufig Zwischenhäfen ansteuern, während Handelsschiffe sich überwiegend in für professionelle Rettungsteams und Rettungshubschrauber nicht erreichbaren Seegebieten bewegen. In der deutschen Nord- und Ostsee besteht durch die Deutsche Gesellschaft zur Rettung Schiffbrüchiger (DGzRS) ein dichtes Netz aus Seenotrettungsbooten und -kreuzern und es ist grundsätzlich möglich, eine medizinisch begründete Evakuierung von Schiffen, Inseln oder anderen Strukturen durchzuführen. Hierbei kommt den seegängigen Hubschraubern, beispielsweise der Marine, aufgrund des Zeitvorteils eine große Bedeutung zu. Diese müssen über eine Rettungswinde verfügen, um Rettungspersonal an Bord zu bringen und im Anschluss den Patienten an Bord übernehmen zu können, da die wenigsten Schiffe über eine Landemöglichkeit verfügen. Neben der notwendigen persönlichen Schutzausrüstung erfordert die Tätigkeit auf Seenotrettungskreuzern und seegängigen Rettungshubschraubern eine spezielle Ausbildung sowie permanentes Training.

Neue Aspekte ergeben sich durch den Bau und Betrieb von Offshorewindparks, für die sich inzwischen betriebliche Luftrettungsdienste etabliert haben.

\section{Vernetzung der Systeme}

Da die Ressourcen für die verschiedenen Bereiche der maritimen Notfallmedizin begrenzt sind, erscheint es sinnvoll, eine umfassende Betrachtung vorzunehmen. Es ist bereits heute so, dass betriebliche Rettungshubschrauber der Offshorewindparks bei Nichtverfügbarkeit der für den SAR-Bereich vorgehaltenen Luftrettungsmittel für maritime Notfalleinsätze angefragt werden. Der Ausbau der Offshorewindenergie wird voranschreiten und auch im Kreuzfahrtbereich ist mit einem weiteren Anstieg der Personenzahlen zu rechnen, sodass in der Zukunft eher mehr als weniger Notfalleinsätze auf See zu verzeichnen sein werden.

In der Folge werden sich die verschiedenen Rettungsmittel der einzelnen Systeme immer wieder an den Schnittstellen begegnen und gegenseitig unterstützen müssen. Es erscheint daher notwendig und geboten, die Bereiche enger zu verzahnen und die Abläufe besser aufeinander abzustimmen.

\section{Entwicklung von Qualitätsstandards} Neben der sehr wichtigen Vernetzung wie beispielsweise am „Runden Tisch Maritime Sicherheitspartnerschaft" der Stiftung Offshore-Windenergie geht es dabei aber auch um die Entwicklung von Standards und Empfehlungen beispielsweise zur Qualifikation des eingesetzten Rettungspersonals sowie für konkrete Notfallsituationen wie zum Beispiel die Hypothermie. Mit einem Anstieg der Notfalleinsätze könnte es zukünftig auch sinnvoll oder gar notwendig werden, die SAR-Hubschrauber notärztlich fest zu besetzen, wie es in anderen europäischen Ländern wie Norwegen bereits der Fall ist. Konsentierte Empfehlungen zur Qualifikation des Rettungspersonals können auch hier sinnvoll sein, um einen einheitlichen Stand zu erreichen.

\section{Wissenschaftliche Aufarbeitung}

\section{der Einsätze}

Eine wissenschaftliche Auswertung des gesamten Einsatzgeschehens könnte eine Unterstützung bieten, um die maritime Notfallmedizin langfristig auf eine belastbare Datengrundlage im Sinne einer evidenzbasierten Medizin zu stellen, wie es beispielsweise die alpine Notfallmedizin vor einigen Jahren bereits begonnen hat. Neben der Untersuchung von Notarzteinsätzen per Schiff oder Hubschrauber könnte ebenso auch die Evaluation des Konzepts für den „SAR-Ersthelfer See“ Erkenntnisse zur kontinuierlichen Verbesserung des Segments der erweiterten Laienhilfe liefern. Bei immer knapperen finanziellen Mitteln wird es zukünftig darauf ankommen, die verschiedenen Einheiten und Konzepte ressourcenschonend einzusetzen. Die DGMM will hierzu durch die Gründung der Arbeitsgruppe „Maritime Notfallmedizin“ einen Beitrag leisten.

\section{Interdisziplinäre \\ Zusammensetzung}

Die Arbeitsgruppe befindet sich derzeit im Aufbau. Aufgrund der vielfältigen Schnittstellen und Besonderheiten ist es für die Erarbeitung belastbarer Ergebnisse zwingend, die AG entsprechend interdisziplinär aufzustellen, sodass Aspekte wie zum Beispiel Allgemeinmedizin, Atemwegsmanagement, Besonderheiten auf Seeschiffen (maritimmedizinische Expertise), funkärztliche Beratung, Hubschrauberrettung, Hypothermie, Kreuzfahrtmedizin, Seenotrettung und Windparks entsprechend fachlich besetzt werden können. Eine Vernetzung zu anderen Fachgesellschaften (z.B. DGAI, DIVI) und Verbänden (z.B. AGNN, BAND) soll die AG mittelfristig als Expertengruppe in der wissenschaftlichen Auseinandersetzung zum Thema „Maritime Notfallmedizin“ erkennbar machen. Wir werden an dieser Stelle weiter über die Arbeit berichten.

Dr. Markus Stuhr, Hamburg; Dr. Jens Kohfahl, Cuxhaven; Dr. Klaus-Herbert Seidenstücker, Hamburg 


\section{Im Mittelpunkt: der an Bord arbeitende Mensch}

50 Jahre nach Errichtung der „Abteilung Schiffahrtsmedizin“ am Hamburger Bernhard-Nocht-Institut für Schiffs-und Tropenkrankheiten und 25 Jahre nach deren Schließung wurde in Hamburg wieder ein forschender Schifffahrtsmediziner zum Privatdozenten ernannt: PD Dr. Marcus Oldenburg, Facharzt für Arbeitsmedizin und Allergologie, leitet seit 2004 die Arbeitsgruppe „Schifffahrtmedizin“ im Hamburger Zentralinstitut für Arbeitsmedizin und Maritime Medizin (Direktor: Prof. Dr. Volker Harth).

In seiner Antrittsvorlesung am 9. Dezember 2015 stand der an Bord arbeitende Mensch „früher und heute“ im Zentrum: Die Auswirkungen der spezifischen Arbeitsumwelt mit ihren physischen, soziokulturellen und psychischen Belastungen, Fragen der Ernährung und der persönlichen Hygiene. Dazu die Entwicklung der medizinischen Versorgung an Bord und aus aktuellem Anlass die psychomentale Belastung durch die Rettung und Betreuung von Flüchtlingen in See. Diese Präsentation zeigt die Notwendigkeit, angesichts der bunten Vielfalt der zeitgemäß firmierten „Maritimen Medizin“, die auf See arbeitenden Frauen und Männer im ärztlichen Fokus zu behalten; die Betreuung von Kreuzfahrtpassagieren bleibt hier ein Randthema.

Markus Oldenburg hat in seinen wissenschaftlichen Arbeiten immer wieder Probleme aufgegriffen, die den Praktikern unter den Nägeln brennen:

- Schabenbefall an Bord,

- Lösemittel in Trinkwassertanks,

- Ernährung von Seeleuten,

- Nutzung von halbautomatischen Defibrillatoren auf Kauffahrteischiffen,

- Schmerzmitteleinsatz auf See,

- medizinische Ausbildung und

- psychomentale Belastung von Seeleuten.

Es ist der Verdienst von Oldenburg, dass er mit großer Hartnäckigkeit Feldforschung zur psychomentalen Belastung in der Schifffahrtsmedizin durchführt, hierbei ein eigenes Instrumentarium entwickelte, die Sozialpartner in den Reedereien und Institutionen überzeugte und selber mit

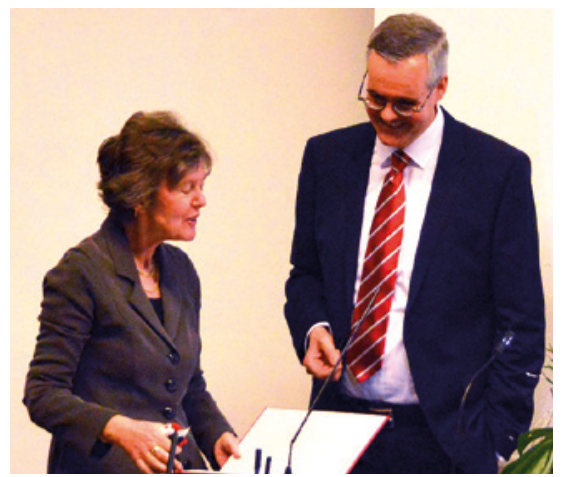

Prof. Dr. Ingrid Moll, UKE, überreicht PD Dr. Marcus Oldenburg die Habilitationsurkunde. Quelle: E. Untiet, ZfAM

seinem Forschungsteam zum Seefahrer wurde. Als Wissenschaftler kennt er nun den Arbeitsplatz See wie kein anderer forschender Arbeitsmediziner.

42 Jahre Pause zwischen den 2 schifffahrtmedizinischen Habilitationen in Hamburg (1973 hatte sich Dr. Hartmut Goethe über „Die Seekinetose“ habilitiert) zeigen, wie schwer es ein Querschnittsfach hat, das eine Fülle von sehr divergierenden Fragestellungen vereinigen muss, sich im akademischen Umfeld zu behaupten. Durch die Initiative der norddeutschen Küstenländer wurde in den 90er Jahren dafür gesorgt, dass die zivile Schifffahrtsmedizin nicht unterging. In dieser Zeit kam es auch zur Gründung unserer Fachgesellschaft, die sich in ihrer Satzung den von Marcus Oldenburg dargestellten Herausforderungen verpflichtet hat.

Mit der Erweiterung des Hamburger Zentralinstituts für Arbeitsmedizin um die maritime Medizin sind jetzt die Voraussetzungen geschaffen, dass die Medizin der Seefahrer in unserem, vom Warentransport über See abhängigen, Land den angemessenen Platz findet. Die Praktiker der Schifffahrtsmedizin erhoffen sich weiterhin eine kontinuierliche und wissenschaftlich fundierte Bearbeitung ihrer täglichen Herausforderungen als Seefahrer- und Reedereiärzte durch das Zentralinstitut für Arbeitsmedizin und Maritime Medizin. Herrn PD Dr. Marcus Oldenburg gratulieren wir herzlich zu seiner Habilitation und wünschen im weiterhin viel Erfolg!

Dr. Clara Schlaich, Hamburg 


\section{Kommunikationsplattform zwischen Wirtschaft, Politik und Forschung}

Die Stiftung OFFSHORE-WINDENERGIE (Stiftung der deutschen Wirtschaft zur Nutzung und Erforschung der Windenergie auf See) wurde im Juli 2005 mit dem Ziel, die Rolle der Offshorewindenergie im Energiemix der Zukunft in Deutschland und Europa zu festigen und ihren Ausbau im Interesse von Umwelt- und Klimaschutz voranzutreiben, gegründet. Als Inhaberin der Genehmigungsrechte des ersten deutschen Offshorewindparks und Testfelds alpha ventus hat die Stiftung die Entwicklung der OffshoreWindenergie-Branche in Deutschland von Anfang an aktiv begleitet und gefördert und sich als überparteiliche, überregionale und unabhängige Einrichtung zur Unterstützung der Windenergie auf See in Deutschland etabliert.

\section{Ziele der Stiftung}

Die Stiftung wirkt als Kommunikationsplattform zwischen Wirtschaft, Politik und Forschung sowie als parteipolitisch unabhängige Interessenvertretung für die Belange der Offshorewindenergie und bündelt die Interessen verschiedenster Akteure. Sie verfügt über vielschichtige Erfahrungen mit der Organisation und Leitung von Moderationsveranstaltungen und Koordination von Projekten und Initiativen.

Kuratoren der Stiftung sind neben Vertretern der zuständigen Ministerien auf Landes- und Bundesebene zahlreiche Akteure aus Branchenverbänden und regionalen Netzwerken, Hersteller von Offshore-Windenergie-Anlagen, Baugesellschaften und Zulieferer, Energieversorgungsunternehmen und Offshoreplaner, Banken, Finanzierungsgesellschaften, Versicherungen und Vertreter der maritimen Wirtschaft.

Die Stiftung unterstützt die verschiedenen politischen Institutionen und Akteure bei dem Ausbau und der Weiterentwicklung der Offshorewindenergie in Deutschland und Europa. Dabei informiert, berät und moderiert sie energiepolitische Gremien. Sie vernetzt die Vertreter der Offshore-WindenergieIndustrie mit der maritimen Wirtschaft. Im Rahmen von Fachgruppen und Ar-

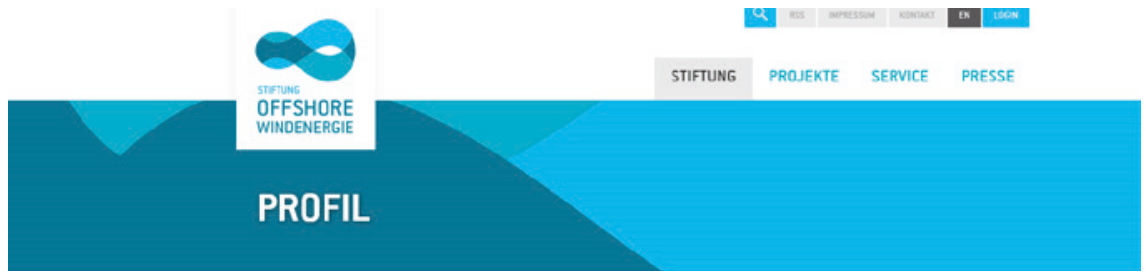

beitskreisen diskutiert sie zusammen mit anderen Branchenvertretern aktuelle Themenfelder der Windenergienutzung auf See und erarbeitet dazu Handlungsempfehlungen und Stellungnahmen.

In Deutschland liegen die meisten Offshorewindparks in der Ausschließlichen Wirtschaftszone (AWZ) und können damit bis zu $120 \mathrm{~km}$ weit von der Küste entfernt liegen. Grund dafür sind die küstennahen Naturschutzgebiete, wie zum Beispiel das Weltnaturerbe Wattenmeer, sowie die ost- und nordfriesischen Inseln. Aber auch die Interessen der Schifffahrt, Fischerei, militärischen Verteidigung und des Tourismus sind bei der Planung der Windparks zu berücksichtigen.

\section{Dialog aller Akteure}

Die Nutzung der Windenergie auf dem Meer stellt hohe Ansprüche an Mensch und Technik. Die Gewährleistung der Sicherheit für das Personal ist durch die Entfernung zur Küste und den teilweise widrigen Bedingungen auf See anspruchsvoll und bedarf der engen Zusammenarbeit aller mitwirkenden Akteure.

Um den Dialog zwischen Bund, Ländern und Unternehmen der Offshorewindenergie sowie der maritimen Wirtschaft zu verbessern, wurde im März 2013 der Runde Tisch „Maritime Sicherheitspartnerschaft" unter Moderation der Stiftung OFFSHORE-WINDENERGIE konstituiert. Die dort behandelten Themen sind vielfältig. Die Teilnehmer befassen sich in den halbjährlich stattfindenden Sitzungen sowie in Arbeitskreisen mit Fragen zu Rettungskonzepten, Telemedizin, Ausbildung von Einsatzkräften und Erste-HilfeAusbildung des Offshore-WindenergiePersonals sowie mit Fragen von staatlicher beziehungsweise unternehmerischer Verantwortung für die Sicherheit in der AWZ. Auf den Sitzungen berichten die Teilnehmer auch über thematisch angelehnte Projekte und Aktivitäten.

Durch den Dialog ist es erstmals gelungen, alle betroffenen staatlichen, privaten und gesellschaftlichen Akteure an einem Tisch zusammenzubringen. Die manchmal auch kontroversen Auseinandersetzungen im Rahmen der maritimen Sicherheitspartnerschaft tragen dazu bei, dass alle relevanten Partner über die Offshore-Windkraft-Entwicklung, F- und E-Projekte, politische Entscheidungen, den Netzausbau, in Arbeitskreisen erzielte Ergebnisse und die jeweiligen wirtschaftlichen Interessen informiert werden sowie aktuelle Probleme gemeinsam lösungsorientiert diskutiert werden können.

Förderung des Runden Tisches gesichert Bis Ende 2015 wurde der Runde Tisch im Rahmen des von der Stiftung koordinierten Projekts „Vernetzung der maritimen Wirtschaft mit der Branche der OffshoreWindenergie“ vom Bundesministerium für Wirtschaft und Energie (BMWi) gefördert. Auf der nationalen maritimen Konferenz am 19./20. Oktober 2015 in Bremerhaven hat der maritime Koordinator der Bundesregierung, der parlamentarische Staatssekretär Uwe Beckmeyer, bei der Vorstellung der Eckpunkte einer Maritimen Agenda 2025 die Fortsetzung der Förderung dieses für die Offshore-Windkraft-Entwicklung wichtigen Projekts zugesagt. Damit ist die Fortführung der Arbeit des Runden Tisches „Maritime Sicherheitspartnerschaft" für die nächsten Jahre gesichert.

Dr. Stephanie Wehkamp, Jörg Kuhbier; Varel

Weitere Informationen im Internet

- www.offshore-stiftung.de/Vernetzung

- www.offshore-stiftung.de/profil 